\title{
Clinical characteristics and treatment outcomes of parathyroid carcinoma: A retrospective review of 234 cases
}

\author{
PEISONG WANG ${ }^{1}$, SHUAI XUE ${ }^{1}$, SHUO WANG $^{1}, \mathrm{ZHI} \mathrm{LV}^{2}$, XIANYING MENG ${ }^{1}$, \\ GUIMIN WANG ${ }^{1}$, WEI MENG ${ }^{1}$, JIA LIU ${ }^{1}$ and GUANG CHEN ${ }^{1}$ \\ ${ }^{1}$ Department of Thyroid Surgery, The First Hospital of Jilin University; ${ }^{2}$ Department of Orthopedics, \\ China-Japan Union Hospital of Jilin University, Changchun, Jilin 130021, P.R. China
}

Received April 4, 2016; Accepted June 27, 2017

DOI: $10.3892 / \mathrm{ol} .2017 .7076$

\begin{abstract}
Parathyroid carcinoma (PC) is one of the rarest known types of cancer and has a moderate prognosis, with estimated 5- and 10-year overall survival rates between $78-85 \%$ and between $49-70 \%$, respectively. To raise awareness of this disease, and to optimize its diagnosis, clinical management and prognosis, the present study retrospectively reviewed 234 cases of PC. A total of 226 cases of PC, which were archived between 1984 and 2015 in the three major databases of the Chinese population, were retrieved and pooled with the 8 cases diagnosed and treated at the Department of Thyroid Surgery of The First Hospital of Jilin University (Changchun, China) between June 2008 and December 2015. The clinicopathological features, diagnosis, surgical procedures and outcomes of these cases of PC were investigated. The review revealed that misdiagnosis has been a considerable issue, with $>80 \%$ of the patients misdiagnosed prior to surgery, and the accuracy of intraoperative diagnosis based on frozen sections was only $15.04 \%$. The use of radical resection as first-line therapy significantly improved the disease-free survival by $\sim 8$ years (log-rank, 20.956; $\mathrm{P}<0.001$ ); and, at relapse, reoperation prolonged patient survival by $\sim 7$ years (log-rank, 35.322; $\mathrm{P}<0.001$ ). Consistently, a Cox proportional hazards analysis indicated that radical resection as a first-line therapy reduced the risk of postoperative recurrence $(\mathrm{P}=0.030)$, and that reoperation following recurrence significantly improved patient survival $(\mathrm{P}=0.030)$. The 5- and 10-year cumulative disease-specific survival rates of the cases of PC were 83 and $67 \%$, respectively. Notably, an increased mortality rate was observed among males with PC compared with female patients with PC. In summary, in the past 32 years (1984-2015), the majority of patients with PC have been misdiagnosed. Performing radical resection
\end{abstract}

Correspondence to: Professor Guang Chen, Department of Thyroid Surgery, The First Hospital of Jilin University, 71 Xinmin Street, Chaoyang, Changchun, Jilin 130021, P.R. China

E-mail: guangchendoc@126.com

Key words: parathyroid carcinoma, thyroid cancer, endocrine cancer, radical resection as the first-line therapy significantly reduces recurrence and improves patient survival time; and, following relapse, subsequent surgery has also been demonstrated to be an effective approach.

\section{Introduction}

Although parathyroid neoplasms are common and often cause primary hyperparathyroidism (pHPT), parathyroid carcinoma (PC) isa rare endocrine malignancy, and accounts for $<1 \%$ of cases of pHPT (1). To date, $>1,000$ cases of PC have been identified in the English literature (2-4); however, there have been no nation-wide large-sample studies in China, and the majority of the available studies are case reports and literature reviews.

The histopathological distinction between benign and malignant parathyroid tumors is often difficult, with up to $50 \%$ of patients presenting with metastases following an initial diagnosis of benign disease $(5,6)$. It is of great importance to raise awareness of $\mathrm{PC}$ in order to reduce its misdiagnosis and to improve its cure rate and prognosis. In the present study, a systematic review of Chinese language literature on PC was performed, and 8 cases of PC diagnosed and treated at the Department of Thyroid Surgery of The First Hospital of Jilin University (Changchun, China) were included.

As PC is resistant to radiotherapy and chemotherapy, en bloc resection of the tumor is usually recommended; however, there is dispute over whether the prophylactic removal of the ipsilateral central compartment lymph nodes and other regional tissues or organs should be performed $(3,6-8)$. Therefore, clinical evaluation, including preoperative examination and intraoperative judgement by the surgeon, are key factors on which the clinical outcome relies. Adjuvant therapy, including chemo-, radio- or anti-parathyroid hormone (PTH) immunotherapy has also been investigated, but the data are limited (3,7,9-11). Therefore, radical resection remains the standard regimen. For recurrent disease, surgery is also recommended as the primary treatment strategy, and the majority of patients with PC require multiple surgeries $(3,7)$.

Throughout the English literature, $>1,000$ cases of PC have been identified (2-4). However, a systematic study on a large Chinese cohort is not available, despite the fact that a large number of cases have been described (12-17). Due to a 
lack of histopathological markers that differentiate malignant from benign parathyroid tumors, up to $50 \%$ of patients with metastases have been misdiagnosed initially with benign disease $(5,6)$. Therefore, it is necessary to raise awareness and improve general understanding of PC.

In the present study, 234 cases of PC archived in three commonly used databases in China, or diagnosed and treated in the Department of Thyroid Surgery of The First Hospital of Jilin University, were reviewed in order to summarize the clinicopathological features relevant to the diagnosis and prognosis of this disease, and the current optimal treatment strategy.

\section{Materials and methods}

Patient selection. A total of three major databases in China, VIP Journal Integration Platform (qikan.cqvip.com), China Knowledge Resource Integrated Database (www.cnki.net), and Wanfang Data (www.wanfangdata.com.cn), were searched (2December 2015) using the key words 'parathyroid cancer' and 'parathyroid tumor'. Subsequent to removing duplicate records or those with incomplete information, a final list of archived cases $(n=226)$ from across China, and which contained detailed information, were obtained. These cases were then combined with the 8 cases of PC that had been diagnosed and managed in The First Hospital of Jilin University, and analyzed in the present retrospective study. All cases included in the present study were in one of the following categories: i) Patients who were diagnosed with parathyroid cancer (18) [including patients who exhibited macroscopic evidence of a parathyroid tumor with a fibrotic capsule and surrounding adhesions, and for whom the histopathological criteria for parathyroid cancer (trabecular pattern, thick fibrous trabeculae, mitotic figures, capsular or vascular invasion and/or lymph node invasion) were subsequently confirmed; patients with a locally invasive tumor; and/or patients with distant metastasis]; or ii) patients who developed local recurrence or nodal and/or distant metastases, and who were initially classified as having benign disease on histology during follow-up $(5,6)$. All the identified cases were from large teaching hospitals affiliated to medical universities in China.

Data extraction. The physical and clinicopathological features that were retrieved for the patients included sex, age, preoperative symptoms and their duration, clinical classification, correct diagnosis vs. incorrect diagnosis, tumor location, type of initial surgery, intraoperative diagnosis, recurrence, follow-up (using patient records), reoperation and outcome. Surgical treatments for primary and recurrent tumors included parathyroidectomy and radical resection. Parathyroidectomy was undertaken to remove the primary tumor, and radical resection also included the ipsilateral parathyroid glands with the surrounding hemithyroid and lymphatic tissues, the unilateral/ipsilateral central compartment and the involved surrounding structures.

Statistical analysis. Data analyses were performed using SPSS software, version 21.0 (IBM Corp., Armonk, NY, USA). Patients who succumbed without $\mathrm{PC}$ recurrence were censored for the disease-specific survival analysis.
The 5- and 10-year disease-free and disease-specific survival rates were estimated using the Kaplan-Meier method, and univariate comparisons of survival time by covariate patterns were evaluated using a log-rank test. Cox proportional hazards analysis was used to study the effects of prognostic factors on the disease-free and disease-specific survival times. $\mathrm{P}<0.05$ was considered to indicate a statistically significant difference.

\section{Results}

$P C$ incidence exhibits a temporally increasing trend. From the three databases in China, 61 PC-relevant medical publications, including 42 case reports and 19 short original articles from between 1984 and 2015, were identified. These publications, in addition to the 8 cases treated at our hospital, included a total of 234 cases of PC from 60 large teaching hospitals affiliated to medical universities in China (Table I). To study the diagnosis and management of PC in China during the past 32 years, the 234 cases were divided into 5 subgroups based on the date of diagnosis (Fig. 1). Patients diagnosed prior to 1995 were pooled, and then those diagnosed during each subsequent 5-year interval (between 1996 and 2015) were grouped. The data indicated an apparent increase in the number of diagnosed PC cases, from 13 cases between 1996 and 2000 to >100 cases between 2011 and 2015 (Fig. 1).

Clinical features. Among the patients, 120 were males and 114 were females, and the male/female ratio was $\sim 1: 1$. The median age at diagnosis was 45.5 years (range, 18-78 years). The average diameter of the tumors was $3.21 \pm 1.16 \mathrm{~cm}$, and the average disease course was 19.58 months in duration, with a maximum time prior to diagnosis of 9 years. There were various subtypes among the study population, which included bone, renal, mixed or other subtypes, and were defined according to the signs and symptoms of pHPT caused by PC (18). 'Bone subtype' refers to bone-associated complications, among which the most common type of bone disease in pHPT is osteitis fibrosacystica (19), which results in pain and occasionally pathological fractures. Other bone diseases associated with pHPT include osteoporosis, osteomalacia and arthritis. 'Renal subtype' refers to kidney stones, nephrocalcinosis and diabetes insipidus (polyuria and polydipsia); these may ultimately lead to renal failure. The 'mixed' subtype consists of both the bone and renal subtypes. Finally, the 'other' subtype consists of nonspecific gastrointestinal, cardiovascular and neuromuscular dysfunction and asymptomatic pHPT. In the present study, various clinical classifications were identified, with the bone and mixed subtypes accounting for 39.74 and $34.62 \%$ of cases, respectively, while 16 cases were recorded as renal and 41 cases as other subtypes. The majority of the 234 PCs were observed in the bilateral lower parathyroid regions, with 114 in the lower right and 91 in the lower left, whereas there were only 29 cases exhibiting tumors in the upper parathyroid region.

Of the 234 patients with PC, a large proportion (191 cases) were misdiagnosed prior to surgery; misdiagnoses included rheumatoid arthritis, urinary stones, bone tumors, pathological fractures, osteoporosis, gout, myeloma, ankylosing spondylitis, osteomyelitis, gastroenteritis, bone tuberculosis, 
Table I. Clinicopathological characteristics of patients with parathyroid carcinoma.

\begin{tabular}{|c|c|}
\hline Variable & Value \\
\hline Total patients, $\mathrm{n}$ & 234 \\
\hline \multicolumn{2}{|l|}{ Sex, $n$} \\
\hline Male & 120 \\
\hline Female & 114 \\
\hline \multicolumn{2}{|l|}{ Age, years } \\
\hline Median & 45.5 \\
\hline Range & $18-78$ \\
\hline \multicolumn{2}{|l|}{ Site of primary tumor, $n$} \\
\hline Left upper & 10 \\
\hline Right upper & 19 \\
\hline Left lower & 91 \\
\hline Right lower & 114 \\
\hline \multicolumn{2}{|l|}{ Pre-diagnosis duration } \\
\hline Mean & 19.58 months \\
\hline Longest & 9 years \\
\hline \multicolumn{2}{|l|}{ Clinical classification, n (\%) } \\
\hline Bone type & $93(39.74)$ \\
\hline Renal type & $16(6.84)$ \\
\hline Mixed & $84(34.62)$ \\
\hline Others & $41(17.52)$ \\
\hline Cases of distant metastasis, $\mathrm{n}(\%)^{\mathrm{a}}$ & 35 \\
\hline Lung & $14(40.00)$ \\
\hline Bone & $9(25.71)$ \\
\hline Mediastinum & $1(2.86)$ \\
\hline Multiple organs & $11(31.43)$ \\
\hline \multicolumn{2}{|l|}{ Initial surgery, number of cases $(\%)$} \\
\hline Radical resection & $141(60.26)$ \\
\hline Parathyroidectomy & $93(39.74)$ \\
\hline \multicolumn{2}{|l|}{ Recurrence } \\
\hline Total, $\mathrm{n}$ & 91 \\
\hline \multicolumn{2}{|l|}{ Following radical resection } \\
\hline Number of cases & 37 \\
\hline Disease-free period & 36 months $^{\mathrm{b}}$ \\
\hline \multicolumn{2}{|l|}{ Following parathyroidectomy } \\
\hline Number of cases & 54 \\
\hline Disease-free period & 24 months $^{\mathrm{b}}$ \\
\hline
\end{tabular}

aPercentage of metastatic cases; ${ }^{b}$ median.

neurosis, weakness, menopausal syndrome, thyroid cancer or pancreatitis. Intraoperative diagnosis performed on the frozen sections did not improve the diagnosis, with only 17 out of the 113 examined being correctly diagnosed. The use of fine-needle aspirate for cytology was prohibited in order to avoid seeding the tumor (5).

Distant metastasis occurred in 35 cases, including 10 patients who exhibited metastatic tumors prior to the first surgery, and did not demonstrate a decrease in serum calcium

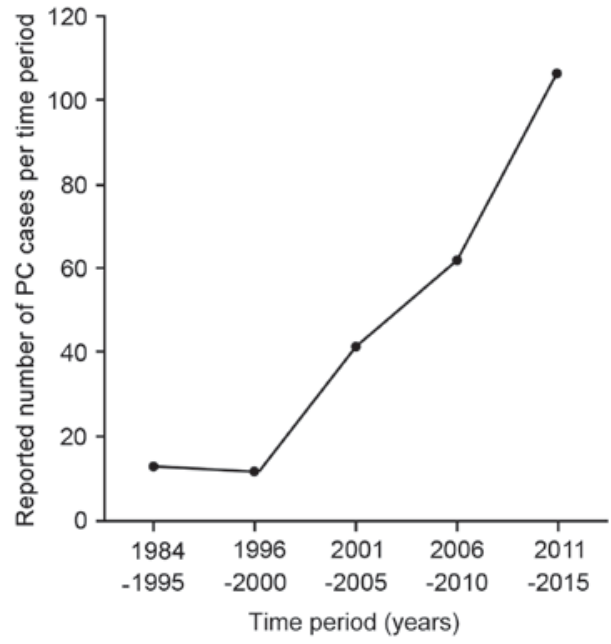

Figure 1. Absolute number of cases of PC reported in China between 1995 and 2015. PC, parathyroid carcinoma.

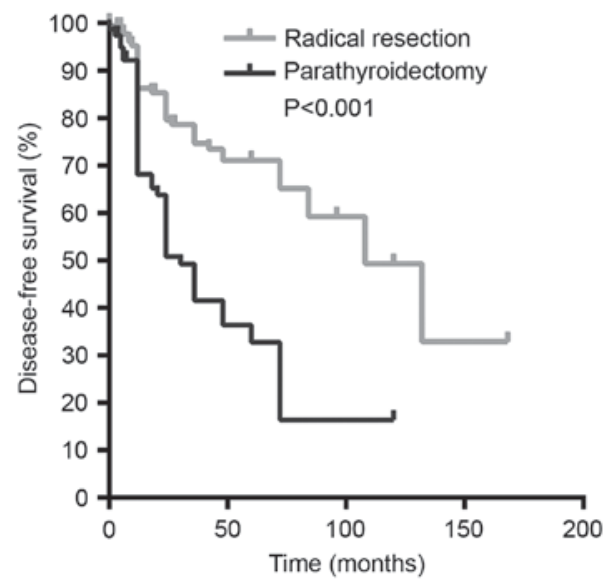

Figure 2. Effect of first-line surgical strategy on disease-free survival in parathyroid carcinoma. Kaplan-Meier survival analysis was performed, and a log-rank test used to compare the patients treated by radical resection $(n=128)$ and by parathyroidectomy $(n=87)$. Radical resection significantly prolonged the disease-free survival time.

following the surgery. Metastatic tumors were identified in the lungs (14 cases), bone ( 9 cases), mediastinum ( 1 case) and multiple organs (11 cases).

Radical resection reduces recurrence and metastasis. Surgery was performed as the first-line therapy in all 234 patients; 141 of them were treated by radical resection, in which the primary tumor, ipsilateral thyroid, surrounding lymph nodes and involved structures were completely removed. The other 93 patients were managed by parathyroidectomy or palliative resection. The advantages of radical resection in reducing the probability of recurrence and prolonging patient survival over other surgical strategies were significant (log-rank, 20.956; $\mathrm{P}<0.001$ ); the median disease-free survival time was increased in patients treated by radical resection compared with those treated by parathyroidectomy (132 vs. 36 months, respectively; Fig. 2; Table II). However, no significant variation in the disease-specific survival between the two surgical strategies was observed (log-rank, 0.021; P=0.885; Fig. 3; Table III). 
Table II. Effect of initial surgery on the probability of recurrence.

\begin{tabular}{lccc}
\hline Surgery type & Total patients, $\mathrm{n}$ & Cases of recurrence, $\mathrm{n}(\%)$ & Pearson's $\chi^{2}$ \\
\hline Parathyroidectomy & 87 & $54(62.07)$ & 23.33 \\
Radical resection & 128 & $37(28.91)$ & $<0.001$ \\
\hline
\end{tabular}

Only the 215 patients with available follow-up data were included.

Reoperation increases survival subsequent to $P C$ recurrence. Of all the patients, 91 experienced recurrent $\mathrm{PC}$, with frequent involvement of the surrounding thyroid gland, trachea, esophagus and strap muscles. Relapse occurred, on average, at 12 months following surgery, but the longest period of time prior to relapse was 132 months. A total of 65 patients among the 91 cases of recurrence underwent additional surgeries ( 8 times in one particular case), and 46 patients responded (hypercalcemia and elevated PTH returned to normal levels). Among all 91 cases of recurrence, 25 patients succumbed to PC, including 11/65 in the surgical group and 14/26 in the non-surgical group (Table IV). The data indicated that multiple surgeries on patients with disease recurrence significantly increased their disease-specific survival time (Fig. 4; log-rank, 35.322; $\mathrm{P}<0.001)$.

Other factors affecting recurrence and survival following $P C$ surgery. The effects of other factors on the disease-free survival were assessed by log-rank test, but no statistically significant associations were identified for age (log-rank, 0.280; $\mathrm{P}=0.596)$, sex (log-rank, 3.233; $\mathrm{P}=0.072$ ) or duration of the preoperative period (log-rank, 0.070; $\mathrm{P}=0.792$ ). In addition, no significant association was observed between the disease-specific survival and age (log-rank, 0.012; $\mathrm{P}=0.912$ ), sex (log-rank $0.074 ; \mathrm{P}=0.786$ ) or preoperative duration of disease (log-rank, 3.438; $\mathrm{P}=0.064$; data not shown).

To further confirm these results, sex, age, preoperative duration, recurrence, reoperation, distant metastasis and radical surgery were assessed for their association with PC recurrence and survival using a multivariate Cox proportional hazards analysis. Radical resection was found to be an independent factor associated with decreased $\mathrm{PC}$ recurrence $(\mathrm{P}=0.030)$. Notably, male sex was associated with an increased mortality rate compared with female sex $(\mathrm{P}=0.040)$, while reoperation was associated with an increased survival rate $(\mathrm{P}=0.030$; Table V).

Prognosis. Follow-up information was available for 215 cases. The patients were followed up for $\leq 15$ years (mean, 3.73 years), and 25 succumbed to PC. The cumulative 5- and 10-year disease-specific survival rates were 83 and $67 \%$, respectively.

\section{Discussion}

The rate of parathyroid cancer misdiagnosis remains high. Since the first study investigating PC (de Quervain, 1904) (19), $>1,000$ cases of this rare cancer have been identified in the literature $(2,3,8,20,21)$. However, the knowledge of this malignancy remains limited, and additional studies

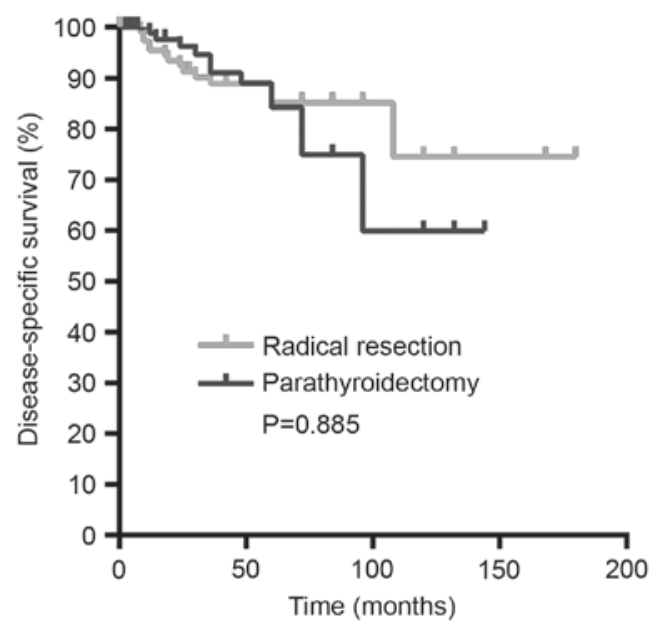

Figure 3. Effect of first-line surgical strategy on disease-specific survival in parathyroid carcinoma. Kaplan-Meier survival analysis was performed, and a log-rank test used to compare the patients treated by radical resection $(n=128)$ and by parathyroidectomy $(n=87)$. No significant effect on the disease-specific survival between surgical strategies was observed.

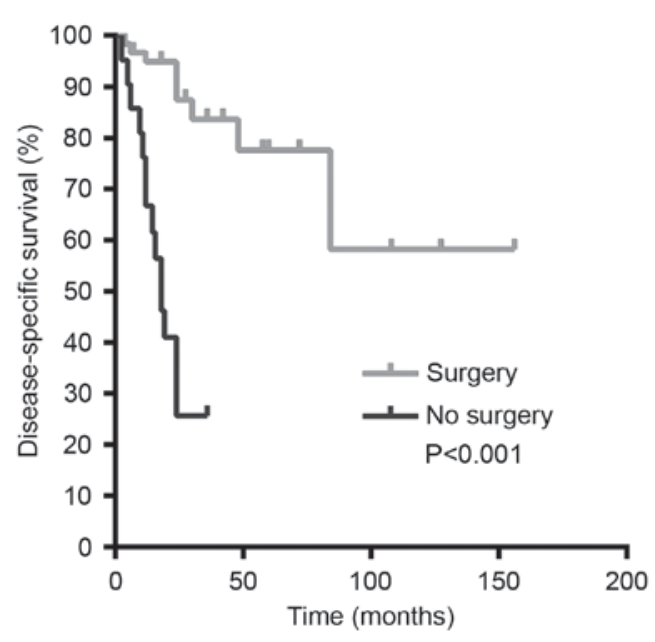

Figure 4. Effect of reoperation following recurrence on disease-specific survival in parathyroid carcinoma. Kaplan-Meier survival analysis was performed, and a log-rank test used to compare the patients who received reoperation following recurrence $(n=65)$ and those who did not receive surgery following recurrence $(n=26)$. Reoperation significantly prolonged the disease-specific survival time.

are required to improve understanding and increase awareness of this disease. In the present study, 234 cases of PC that were diagnosed and managed in 60 hospitals across China were collectively analyzed to screen for clinicopathological features that may potentially assist in the diagnosis and 
Table III. Effect of initial surgeries on the disease-specific survival.

\begin{tabular}{lccc}
\hline Surgery type & Total patients, $\mathrm{n}$ & Mortalities, $\mathrm{n}(\%)$ & Pearson's $\chi^{2}$ \\
\hline Parathyroidectomy & 87 & $9(10.34)$ & 0.23 \\
Radical resection & 128 & $16(12.50)$ & 0.885 \\
\hline
\end{tabular}

Only the 215 patients with available follow-up data were included.

Table IV. Effects of surgical treatment following recurrence $(n=91)$ on patient outcomes.

\begin{tabular}{lccc}
\hline Treatment & Total patients, $n$ & Mortalities, n $(\%)$ & Pearson's $\chi^{2}$ \\
\hline Surgical & 65 & $11(16.92)$ & 12.71 \\
Non-surgical & 26 & $14(53.85)$ & $<0.001$ \\
\hline
\end{tabular}

Table V. Multivariate Cox proportional hazards analysis.

\begin{tabular}{|c|c|c|c|c|c|c|c|}
\hline \multirow[b]{2}{*}{ Variable } & \multirow[b]{2}{*}{$\mathrm{B}$} & \multirow[b]{2}{*}{ SE } & \multirow[b]{2}{*}{ Wald } & \multirow[b]{2}{*}{ P-value } & \multirow[b]{2}{*}{$\operatorname{Exp}(B)$} & \multicolumn{2}{|c|}{$95 \%$ CI for $\operatorname{Exp}(\mathrm{B})$} \\
\hline & & & & & & Lower & Upper \\
\hline \multicolumn{8}{|l|}{ Survival } \\
\hline Sex & -1.61 & 0.81 & 3.99 & 0.04 & 0.20 & 0.04 & 0.96 \\
\hline Age & 0.01 & 0.03 & 0.08 & 0.77 & 1.01 & 0.95 & 1.07 \\
\hline Preoperative duration & -0.25 & 0.18 & 1.88 & 0.17 & 0.78 & 0.55 & 1.11 \\
\hline Recurrence status & -2.44 & 131.41 & 0.00 & 0.98 & 0.08 & 0.00 & 184.90 \\
\hline Reoperation & -2.64 & 1.25 & 4.46 & 0.03 & 0.07 & 0.01 & 0.826 \\
\hline Distant metastasis & 12.27 & 100.78 & 0.02 & 0.90 & 316.12 & 0.00 & 325.28 \\
\hline Radical surgery & -0.09 & 0.67 & 0.02 & 0.88 & 0.90 & 0.24 & 3.38 \\
\hline \multicolumn{8}{|l|}{ Recurrence } \\
\hline Sex & -0.27 & 0.36 & 0.59 & 0.44 & 0.76 & 0.37 & 1.52 \\
\hline Age & 0.01 & 0.01 & 0.04 & 0.84 & 1.00 & 0.97 & 1.03 \\
\hline Preoperative duration & -0.14 & 0.13 & 1.23 & 0.26 & 0.86 & 0.67 & 1.11 \\
\hline Radical surgery & -0.74 & 0.35 & 4.66 & 0.03 & 0.47 & 0.23 & 0.93 \\
\hline
\end{tabular}

B, partial regression coefficients; SE, standard error of the partial regression coefficients; Wald, $\chi^{2}$ value; Exp (B), relative hazard ratio; CI, confidence interval.

treatment of this malignancy, and to identify optimal therapeutic strategies.

The data obtained from the present study indicated a marked increase in the number of PC cases identified in China over 32 years (Fig. 1). This may suggest the rising incidence of PC with industrialization, as documented for other types of cancer $(22,23)$, but may be due to advances in diagnosis. The routinization of blood calcium testing allows incidental and symptom-independent diagnosis of hyperparathyroidism (24). Apart from an excessive secretion of parathyroid hormone and hypercalcemia, that clinical features that are indicative of PC include the following: A palpable neck mass $(>3 \mathrm{~cm})$; hyperparathyroidism concomitant with jaw tumor; hoarseness and/or neck pain; an albumin-corrected calcium level $>3 \mathrm{mmol} / \mathrm{l}$; infiltration and/or calcification visible on neck ultrasound;
PTH levels $>3$ times the upper limit of normal; local invasion and metastasis observed intraoperatively or on sestamibi parathyroid scintigraphy and/or computed tomography scans; significant bone and kidney involvement; and a family history of hyperparathyroidism-jaw tumor syndrome (24). The data from the present study suggested that intraoperative diagnosis on frozen sections did not assist in distinguishing benign from malignant disease, which was consistent with a previous study (25). A definitive diagnosis requires evidence of vascular invasion, perineural invasion, capsular penetration into the adjacent structures, and metastases $(26,27)$.

As the genetic features of the 234 patients in the present study were not recorded and not available in the databases, the molecular mechanisms driving tumorigenesis remain unclear. In previous studies, $>70 \%$ of cases of sporadic PC 
were identified to have mutations in the cell division cycle 73 (CDC73) gene $(13,14,28)$. Immunohistochemically, the loss of the expression of parafibromin (encoded by $C D C 73$ ), retinoblastoma protein, $\mathrm{p} 27, \mathrm{Bcl}-2-\alpha$, mouse double minute 2 homolog and adenomatous polyposis coli gene, and elevation of galectin-3, tumor protein 53 and proliferation marker protein $\mathrm{Ki}-67$ ( $>5 \%$ ) have been demonstrated (24). In particular, parafibromin, galectin-3 and Bcl-2- $\alpha$ are the most useful ancillary biomarkers (29).

The present study indicates that initial radical resection is essential to reduce the recurrence of PC. Due to the difficulties in the diagnosis of $\mathrm{PC}$, the intraoperative assessment by the surgeon of the local invasion of surrounding structures determines whether the lesion is malignant, and what the optimal surgical procedure is. Therefore, the adjacent structures such as the ipsilateral thyroid lobe and thymus, paratracheal alveolar tissue and lymph nodes, require careful inspection and removal, if necessary, to minimize the chance of tumor cells remaining within the body $(7,24,30)$. In general, radical resection of the tumor with safe margins is the first-line therapy of choice (31). Among the 234 patients in the present study, $60.26 \%$ underwent radical resection, and these patients experienced a lower rate of recurrence compared with those who underwent parathyroidectomy only (Table II; Fig. 2). Failure to perform radical resection was an independent risk factor for PC recurrence (Table V). However, radical resection did not confer an overall survival advantage for the patients compared with parathyroidectomy (Table III; Fig. 3). This is consistent with previous results in which no difference in survival was observed between simple excision and en bloc resection $(7,32)$. However, local resection becomes problematic when tumor spillage and parathyromatosis are involved (30). Similarly, Koea and Shaw (6) demonstrated that en bloc resection of the tumor at first presentation was associated with an improved local disease control, and their data also indicated that radical resection significantly improved long-term survival.

Recurrence of PC often occurs around 3 years after the first surgery $(7,30)$. Although the resection of metastatic tumors rarely offers the possibility of cure, removal of the recurrent or metastatic tumors relieves suffering. Patients with PC undergo, on average, two to three surgeries during the course of their disease (33), and extensive neck and mediastinal exploration are recommended in cases of local recurrence. Surgical resection is also recommended to control hypercalcemia, particularly for those with local recurrence or limited distant metastases (34).

Lung metastasis is common in PC, and the resection of pulmonary metastases can result in long-term remission of PC $(1,30,35,36)$. When metastasis is identified toward the lateral neck lymph nodes, dissection of the level II-V lymph nodes should be performed. In cases of mediastinal lymph node or limited lung involvement, laparoscopic or open surgery should be considered $(7,24,30)$. In the present study, a total of 329 parathyroid surgeries were performed on the 234 patients with PC (an average of 1.41 surgeries per patient). Reoperation significantly prolonged the survival of the patients with recurrence (Table IV; Fig. 4). Male sex and lack of reoperation following recurrence were independent negative risk factors for patient survival (Table V).
In summary, in the present study, 234 PC cases during a 32-year period in China were collected, and their diagnosis, treatment and clinical outcomes were analyzed. The data suggested that PC has primarily been misdiagnosed in China due to its sporadic occurrence and the lack of awareness. Radical resection as the first-line therapy significantly reduced the chance of tumor recurrence. Following recurrence, reoperation represents an ideal approach to inhibit cancer progression and improve the clinical outcome.

\section{Acknowledgements}

The present study was financially supported by the Department of Health, Jilin Province, China, awarded to Guang Chen (grant no. 2010Z100).

\section{References}

1. Shane E: Clinical review 122: Parathyroid carcinoma. J Clin Endocrinol Metab 86: 485-493, 2001.

2. Mori H, Okada Y, Arao T and Tanaka Y: Case of parathyroid carcinoma with a highly aggressive clinical course. J UOEH 36: 243-249, 2014.

3. Sadler C, Gow KW, Beierle EA, Doski JJ, Langer M, Nuchtern JG, Vasudevan SA and Goldfarb M: Parathyroid carcinoma in more than 1,000 patients: A population-level analysis. Surgery 156: 1622-1630, 2014

4. Asare EA, Sturgeon C, Winchester DJ, Liu L, Palis B, Perrier ND, Evans DB, Winchester DP and Wang TS: Parathyroid carcinoma: An update on treatment outcomes and prognostic factors from the national cancer data base (NCDB). Ann Surg Oncol 22: 3990-3995, 2015.

5. Marcocci C, Cetani F, Rubin MR, Silverberg SJ, Pinchera A and Bilezikian JP: Parathyroid carcinoma. J Bone Miner Res 23: 1869-1880, 2008.

6. Koea JB and Shaw JH: Parathyroid cancer: Biology and management. Surg Oncol 8: 155-165, 1999

7. Busaidy NL, Jimenez C, Habra MA, Schultz PN, El-Naggar AK, Clayman GL, Asper JA, Diaz EM Jr, Evans DB, Gagel RF, et al: Parathyroid carcinoma: A 22-year experience. Head Neck 26: 716-726, 2004

8. Givi B and Shah JP: Parathyroid carcinoma. Clin Oncol (R Coll Radiol) 22: 498-507, 2010.

9. Mohebati A, Shaha A and Shah J: Parathyroid carcinoma: Challenges in diagnosis and treatment. Hematol Oncol Clin North Am 26: 1221-1238, 2012.

10. Schott M, Feldkamp J, Schattenberg D, Krueger T, Dotzenrath C, Seissler $\mathbf{J}$ and Scherbaum WA: Induction of cellular immunity in a parathyroid carcinoma treated withtumor lysate-pulsed dendritic cells. Eur J Endocrinol 142: 300-306, 2000.

11. Bradwell AR and Harvey TC: Control of hypercalcaemia of parathyroid carcinoma by immunisation. Lancet 353: 370-373, 1999.

12. Wang L, Han D, Chen W, Zhang S, Wang Z, Li K, Gao Y, Zou S and Yang A: Non-functional parathyroid carcinoma: A case report and review of the literature. Cancer Biol Ther 16: 1569-1576, 2015.

13. Zhang J, Tong F, Zhong W and Li S: A case report of anti-parathyroid hormone immunotherapy for parathyroid carcinoma. J Diabetes Endocrinol 2: 36-40, 2011.

14. Siu WK, Law CY, Lam CW, Mak CM, Wong GW, Ho AY, Ho KY, Loo KT, Chiu SC, Chow LT, et al: Novel nonsense CDC73 mutations in Chinese patients with parathyroid tumors. Fam Cancer 10: 695-699, 2011.

15. Zhao J, Hu Y, Liao Q, Niu Z, Xing X, Xia W and Zhao Y: Gene identification of potential malignant parathyroid tumors phenotype in Chinese population. Endocr J 61: 597-605, 2014.

16. Cao J, Chen C, Wang QL, Xu JJ and Ge MH: Parathyroid carcinoma: A report of six cases with a brief review of the literature. Oncol Lett 10: 3363-3368, 2015.

17. McClenaghan F and Qureshi YA: Parathyroid cancer. Gland Surg 4: 329-338, 2015.

18. Harari A, Waring A, Fernandez-Ranvier G, Hwang J, Suh I, Mitmaker E, Shen W, Gosnell J, Duh QY and Clark O: Parathyroid carcinoma: A 43-year outcome and survival analysis. J Clin Endocrinol Metab 96: 3679-3686, 2011. 
19. Dąbrowska A, Tarach J and Zwolak A: Primary hyperparathyroidism due to parathyroid cancer-a diagnostic and management challenge. Endokrynol Pol 66: 150, 157-167, 2015.

20. Talat $\mathrm{N}$ and Schulte KM: Clinical presentation, staging and long-term evolution of parathyroid cancer. Ann Surg Oncol 17: 2156-2174, 2010

21. Villar-del-Moral J, Jiménez-García A, Salvador-Egea P, Martos-Martínez JM,Nuño-Vázquez-Garza JM,Serradilla-MartínM, Gómez-Palacios A, Moreno-Llorente P, Ortega-Serrano J and de la Quintana-Basarrate A: Prognostic factors and staging systems in parathyroid cancer: A multicenter cohort study. Surgery 156: 1132-1144, 2014.

22. Schwartz GG and Sahmoun AE: Ovarian cancer incidence in the United States in relation to manufacturing industry. Int J Gynecol Cancer 24: 247-251, 2014.

23. Stevens RG: Working against our endogenous circadian clock: Breast cancer and electric lighting in the modern world. Mutat Res 680: 106-108, 2009.

24. Duan K and Mete Ö: Parathyroid Carcinoma: Diagnosis and Clinical Implications. Turk Patoloji Derg 31 (Suppl 1): S80-S97, 2015.

25. Kassahun WT and Jonas S: Focus on parathyroid carcinoma. Int J Surg 9: 13-19, 2011

26. DeLellis RA: Parathyroid carcinoma: An overview. Adv Anat Pathol 12: 53-61, 2005.

27. Okamoto T, Iihara M, Obara $\mathrm{T}$ and Tsukada T: Parathyroid carcinoma: Etiology, diagnosis, and treatment. World J Surg 33: 2343-2354, 2009.

28. Gill AJ: Understanding the genetic basis of parathyroid carcinoma. Endocr Pathol 25: 30-34, 2014.
29. Erovic BM, Harris L, Jamali M, Goldstein DP, Irish JC, Asa SL and Mete O: Biomarkers of parathyroid carcinoma. Endocr Pathol 23: 221-231, 2012.

30. Kebebew E, Arici C, Duh QY and Clark OH: Localization and reoperation results for persistent and recurrent parathyroid carcinoma. Arch Surg 136: 878-885, 2001.

31. Munson ND, Foote RL, Northcutt RC, Tiegs RD, Fitzpatrick LA Grant CS, van Heerden JA, Thompson GB and Lloyd RV: Parathyroid carcinoma: Is there a role for adjuvant radiation therapy? Cancer 98: 2378-2384, 2003.

32. Owen RP, Silver CE, Pellitteri PK, Shaha AR, Devaney KO, Werner JA, Rinaldo A and Ferlito A: Parathyroid carcinoma: A review. Head Neck 33: 429-436, 2011.

33. Obara T and Fujimoto Y: Diagnosis and treatment of patients with parathyroid carcinoma: An update and review. World J Surg 15: 738-744, 1991.

34. Qiu ZL, Wu CG, Zhu RS, Xue YL and Luo QY: Unusual case of solitary functioning bone metastasis from a 'parathyroid adenoma': Imagiologic diagnosis and treatment with percutaneous vertebroplasty-case report and literature review. J Clin Endocrinol Metab 98: 3555-3561, 2013.

35. Hundley JC, Albertson DA, Bradley RF and Levine EA: Resection of pulmonary metastasis from parathyroid carcinoma. Am Surg 69: 779-783, 2003.

36. Iihara M, Okamoto T, Suzuki R, Kawamata A, Nishikawa T, Kobayashi $\mathrm{M}$ and Obara T: Functional parathyroid carcinoma: Long-term treatment outcome and risk factor analysis. Surgery 142: 936-943.e1, 2007. 